\title{
High-throughput sequencing
} technology reveals that continuous cropping of American ginseng results in changes in the microbial community in arable soil

\author{
Linlin Dong, Jiang Xu, Lianjuan Zhang, Juan Yang, Baosheng Liao, Xiwen Li and Shilin Chen *
}

\begin{abstract}
Background: American ginseng (Panax quinquefolius L.) is renowned worldwide for its eutherapeutic effects. The replantation of American ginseng usually fails due to problems associated with continuous cropping. An imbalance in the microbial community is thought to be responsible for this, but the overall changes in microbial communities under a continuous cropping system are unclear.
\end{abstract}

Methods: This study used quantitative polymerase chain reaction combined with high-throughput sequencing methods to confirm changes in a microbial community under continuous cropping of American ginseng.

Results: Copy numbers of bacteria and fungi significantly declined by 47.7 and $45.5 \%$, respectively, upon American ginseng cropping over 3 years. A total of 66,391 classified sequences were obtained from high-throughput sequencing analyses of 165 and $18 \mathrm{~S}$ rRNA in six soil samples. A decline in bacterial diversity and an increase in fungal diversity were observed in the continuous cropping soils of American ginseng compared to those of traditional crops. Compared with soils used for traditional crops, the relative abundance of bacterial and fungal groups changed in soils subjected to continuous cropping with American ginseng.

Conclusions: Our results revealed that the diversity and composition of soil bacterial and fungal communities changed in the continuous cropping of American ginseng compared to those of traditional crops. Those data provided comprehensive insight into microbial communities at the agro-ecosystem scale and contributed to the understanding of micro-ecological environments in the rhizosphere of medicinal plants.

Keywords: Panax quinquefolius L., Continuous cropping system, Bacterial community, Fungal community, High-throughput sequencing technology

\section{Background}

American ginseng (Panax quinquefolius L.) is a remarkable medicine that is renowned globally for its eutherapeutic effects [1]. It is a perennial plant that is usually continuously cultivated for 3 years in China. However, the replantation of American ginseng usually fails due to problems associated with continuous cropping [2]. These problems include deterioration of the physicochemical

*Correspondence: slchen@icmm.ac.cn

Institute of Chinese Materia Medica, China Academy of Chinese Medical Sciences, Beijing 100700, China properties of soil, autotoxicity, and changes in the soil microbial community $[3,4]$. Among these, imbalances in soil microbial communities were shown to be the pivotal problem with continuous cropping system [5].

Soil microbial communities are abundant and play important roles in the biochemical cycles of terrestrial ecosystems [6]. The diversity and composition of soil microbial communities are critical for maintaining soil health and quality $[7,8]$. Changes in the diversity and composition of microbial community are related to shifts in a series of biotic and/or abiotic factors such as the cropping system, plant species, and soil type $[9,10]$. 
Many studies have reported that cropping systems have significant effects on the diversity and composition of microbial communities [5, 11]. For example, based on phospholipid fatty-acid analyses, American ginseng cultivation was shown to cause a decline in metabolism function and a shift in the structure of soil microbial communities in the rhizosphere [12]. Therefore, changes in the microbial community can affect soil micro-ecology and thus plant growth. High-throughput sequencing data revealed that fungal diversity declined and changes of microbial community were occurred in the soils of Panax notoginseng continuous cropping for 3 years [13]. However, few studies have simultaneously reported on the changes in bacterial and fungal communities that are associated with the continuous cropping of American ginseng.

In this study, our objective was to detect changes in the diversity and composition of bacterial and fungal communities under a continuous cropping system of American ginseng. The obtained results should be useful for understanding the effects of cropping systems on soil micro-ecology, contribute to the cultivation of medicinal plants, and improve the sustainable development of the medicinal industry.

\section{Methods}

\section{The experiment and soil collection}

This experiment was conducted in Huairou, Beijing $\left(40^{\circ} 300^{\prime} \mathrm{N}, 116^{\circ} 600^{\prime} \mathrm{E}\right)$, which is one of the main areas of American ginseng production in China. American ginseng was cultivated on traditional farmlands, in which mainly maize had been planted for more than 30 years. In the process of maize cultivation, Organic fertilizer $\left(3.0 \mathrm{~kg} \mathrm{~m}^{-2}\right)$ was applied as base fertilizer. The soil moisture content was mediated according to the developmental stages of maize. American ginseng was cultivated following the standard operating procedures of Good Agricultural Practice [14, 15]. Organic fertilizer $\left(2.5 \mathrm{~kg} \mathrm{~m}^{-2}\right)$ was added in October each year. The soil moisture content is $40-50 \%$. Field plots in our plantation were arranged in a fully randomized block design with three replicate plots $(1.5 \times 30 \mathrm{~m})$. Six plants grown for 3 years were chosen from one plot of American ginseng farmland (AGF) and their soil samples in the rhizosphere were collected [16]. Soil samples from maize rhizospheres were used as controls (traditional farmland: TF). Six soil samples from one plot were combined to form a single sample. Those samples were AGF1, AGF2, AGF3, TF1, TF2 and TF3. In total, six samples were gathered, homogenized by being passed through a $2-\mathrm{mm}$ sieve, and stored at $-80^{\circ} \mathrm{C}$ until subjected to further processing for the experiments. The characteristics of these soil samples are shown in Additional file 1: Table S1.

\section{DNA extraction and quantitative polymerase chain reaction (QPCR)}

Total DNA was extracted from freeze-dried soil using the MoBio Powersoil Kit (MoBio, Inc., Carlsbad, CA, USA) based on the manufacturer's instructions. Total DNA was used to analyze the changes in the microbial communities between American ginseng and maize soils. The flow chart of the experimental procedure was showed in Fig. 1. Copy numbers of bacterial and fungal small rRNA subunit genes were calculated as described by Fierer et al. [17] and Rousk et al. [18]. Briefly, fungal gene fragments were amplified using the 5.8S/ITS1f primer pair and bacterial gene fragments were amplified using the Eub338/Eub518 primer pair. Quantitative PCR was performed in a volume of $25 \mu \mathrm{L}$ with the $2 \times$ SYBR Green PCR Master Mix (Takara Bio, Shiga, Japan) using an ABI7500 Fast Real-Time PCR system (Applied Biosystems, Foster City, CA, USA). Double-distilled water was used in the controls instead of template DNA. Copy numbers of bacterial and fungal genes were determined using a regression equation for each assay. Cycle threshold $(C t)$ values were obtained from the known copy numbers in the standards.

\section{PCR amplification and lon Torrent ${ }^{\mathrm{TM}}$ Sequencing}

For each sample, the gene sequences of $16 \mathrm{~S}$ rRNA and $18 \mathrm{~S}$ rRNA were respectively amplified using conserved bacterial primers [17] and conserved fungal primers $817 \mathrm{~F} / 1196 \mathrm{R}$ [18]. The forward and reverse primers each contained a 10-bp barcode, as shown in Additional file 1: Table S2. PCR was carried out in quadruplicate in $25-\mu \mathrm{L}$ reaction mixtures, each containing $1 \mu \mathrm{L}(10 \mathrm{mM})$ of each forward and reverse primer, $2 \mu \mathrm{L}$ of template DNA, $2.5 \mu \mathrm{L}$ of reaction buffer, $2.5 \mu \mathrm{L}$ of dNTP, and

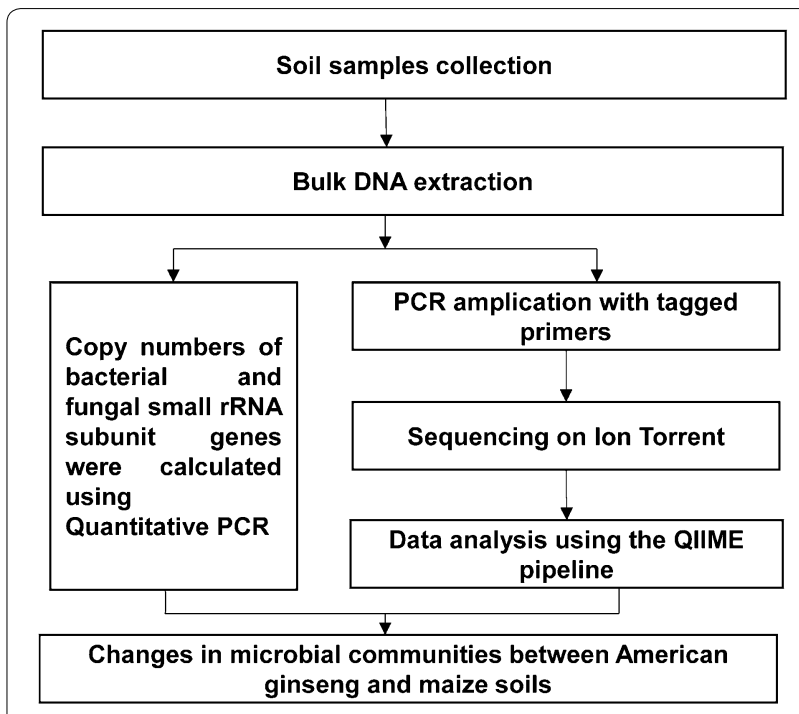

Fig. 1 The flow chart of the experimental procedure 
$0.2 \mu \mathrm{L}$ of Taq DNA recombinant polymerase (Takara, Otsu, Japan). Samples were denatured at $94{ }^{\circ} \mathrm{C}$ for $3 \mathrm{~min}$ and then amplified using 28 cycles at $94{ }^{\circ} \mathrm{C}$ for $45 \mathrm{~s}, 50{ }^{\circ} \mathrm{C}$ for $30 \mathrm{~s}$, and $72{ }^{\circ} \mathrm{C}$ for $60 \mathrm{~s}$. A final extension of $10 \mathrm{~min}$ was added at the end of the program. Negative controls (no templates) were included to check for primer or sample DNA contamination. PCR products were excised from 1\% agarose gel, purified using the MinElute Gel Extraction Kit (Qiagen, Valencia, CA, USA), and quantified using a Quant-iT PicoGreen dsDNA Assay Kit (Invitrogen, Carlsbad, CA, USA). The resulting amplicons were pooled in equimolar ratios. Sequencing was performed on an Ion Torrent ${ }^{\mathrm{TM}}$ Personal Genome Machine using the Ion Xpress Template Kit (Life Technologies, Carlsbad, CA, USA) and the Ion 314 chip (Life Technologies) following the manufacturer's protocol.

\section{Data analysis}

Data were processed as described by the QIIME pipeline [19]. In brief, bacterial and fungal sequences were trimmed and assigned to different samples based on their barcodes. Sequences were binned into operational taxonomic units (OTUs) at the $97 \%$ similarity level. Representative sequence alignments were generated through assignment with PyNAST, sequence alignment was performed to remove gaps, and locations known to be excessively variable were filtered [20]. The filtered alignment sequences were then used to build a phylogenetic tree using FastTree [21]. The taxonomic identities of bacteria and fungi were respectively determined using the RDP [22] and Sliva schemes [23]. Alpha diversities of bacterial and fungal communities were determined to assay phylogenetic diversity, along with Shannon indices $\left(H^{\prime}\right)$, Chao 1, and observed species by a modified version of the procedure described by Caporaso et al. [19]. Pairwise distances between communities were determined using Weighted Unifrac values, and the results of microbial communities were visualized in a principal coordinate analysis (PCoA) matrix.

\section{Statistical analyses}

SPSS version 16.0 was used for statistical analyses of the diversity and relative abundance of soil microbial communities (SPSS Inc., Chicago, IL, USA). The parameters were calculated for all replicates and subjected to an analysis of variance by one-way ANOVA. The value of each bar represents the mean $\pm \mathrm{SD}$ of $n=3$. Mean values were reported as significant or non-significant by paired $t$-tests $(P<0.05)$.
The Minimum Standards of Reporting Checklist contains details of the experimental design, and statistics, and resources used in this study (Additional file 2).

\section{Results \\ Estimation of the ratio of fungi to bacteria by quantitative PCR}

The copy numbers of bacterial and fungal communities had significantly declined by 47.7 and $45.5 \%$, respectively, in soils subjected to continuous cropping with American ginseng compared with those of traditional cropping system (Fig. 2a, b). Additionally, the ratio of fungi to bacteria showed an increasing trend in soils of American ginseng compared to those of traditional crops (maize) (Fig. 2c).

\section{Bacterial diversity was lower in soils used for cultivating American ginseng}

A total of 45,788 sequences were obtained by highthroughput sequencing analyses of $16 \mathrm{~S}$ rRNA gene sequences, with an average of 7631 sequences per sample (range 5812-9458; most common length range 220229 bp) (Additional file 1: Table S3). Chao 1, observed species, phylogenetic diversity, and Shannon index showed decreasing trends in soils used for American ginseng cultivation compared with those in the traditional cropping system (Fig. 3).

\section{Fungal diversity was higher in soils used for cultivating American ginseng}

A total of 20,603 sequences were obtained in highthroughput sequencing analyses of $18 \mathrm{~S}$ rRNA gene sequences (Additional file 1: Table S3). The average number of sequences per sample was 3433 (range 14545563; most common length range 225-282 bp). Chao 1 , observed species, phylogenetic diversity, and Shannon index showed increasing trends in soils used to cultivate American ginseng compared with those of traditional cropping system (Fig. 4).

\section{Changes of bacterial composition in soils used to cultivate American ginseng}

Bacterial composition differed significantly between soils used for American ginseng cultivation and those of maize cultivation (Fig. 5). PCoA ordination showed variations in the bacterial community among soils used for cultivating American ginseng and for maize (Fig. 5a). The first principal component (35.8\% contribution) and the second principal component axes (24.9\% contribution) differentiated the bacterial communities in soils used for cultivating American ginseng and maize. 

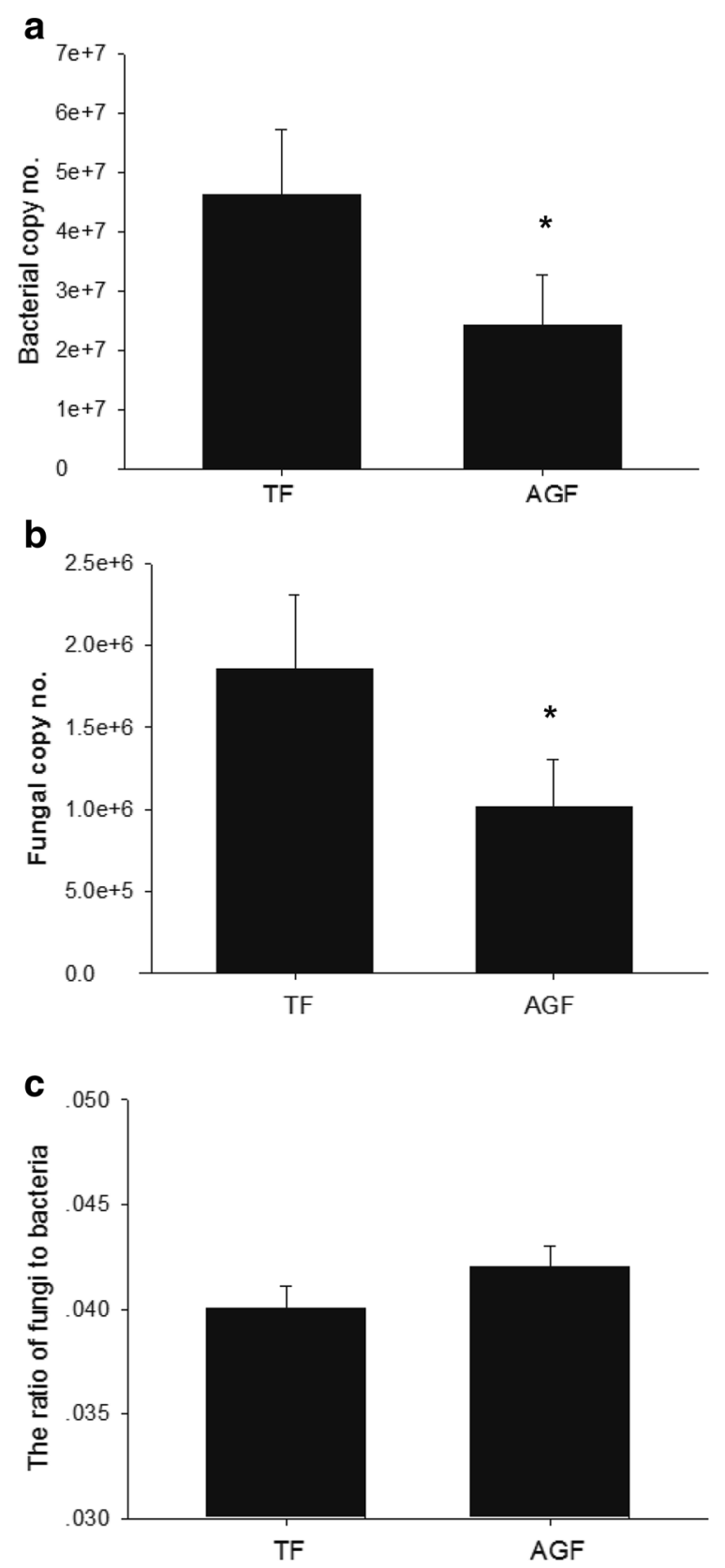

Fig. 2 Copy numbers of bacteria (a), fungi (b) and the ratio of fungi to bacteria (c). TF and AGF refer to soil samples from traditional farmland and American ginseng farmland, respectively. The value of each bar represents the mean $\pm S D$ of $n=3$. An asterisk denotes a significant difference between TF and AGF at $P<0.05$

The distribution of bacterial groups at the phylum level differed between the American ginseng and maize soils (Fig. 5b). Specifically, the relative abundances of Acidobacteria, Alphaproteobacteria, Gammaproteobacteria, Chloroflexi, Betaproteobacteria, Planctomycetes, OD1, AD3, and Verrucomicrobia were higher in soils used to cultivate American ginseng than in those used for maize cultivation. Meanwhile, the relative abundances of Actinobacteria, Bacteroidetes, Deltaproteobacteria, Gemmatimonadetes, Firmicutes, and Nitrospirae were lower in the American ginseng soils.

\section{Changes in the fungal composition of soils used for American ginseng cultivation}

Fungal compositions also differed between American ginseng and maize soils (Fig. 6). These compositions showed variations based on PCoA ordination analyses (Fig. 6a). The first principal component (57.3\% contribution) and second principal component axes $(12.7 \%$ contribution) differentiated the fungal composition of American ginseng and maize soils.

In addition, the distribution of fungal communities varied between soils used for cultivating American ginseng and those of maize at the phylum level (Fig. 6b). Compared with soils used for maize cultivation, the relative abundances of Pezizomycotina, Chytridiomycota, Blastocladiomycota, and Glomeromycota were lower and the relative abundances of Basidiomycota and Saccharomycotina were higher in the American ginseng soils.

\section{The relative abundance of bacterial groups changed in soils of American ginseng cultivation}

Bacterial groups also differed between the American ginseng and maize soils at the levels of order and family (Fig. 7; Additional file 1: Tables S4, S5). The relative abundances of Acidimicrobiales, Actinomycetales, Gaiellales, Solirubrobacterales, Sphingobacteriales, Bacillales, Nitrospirales, Sphingomonadales, Burkholderiales, Myxococcales, and Syntrophobacterales declined by $0.1-49.6 \%$ in soils used to cultivate American ginseng compared with those of maize cultivation (Fig. 7a). However, the relative abundances of Acidobacteriales, Solibacterales, Rhizobiales, Rhodospirillales, Pseudomonadales, and Xanthomonadales increased by $8.1-160 \%$. In soils used for American ginseng cultivation, the relative abundances of Micrococcaceae, Nocardioidaceae, Gaiellaceae, Chitinophagaceae, Flammeovirgaceae, Flexibacteraceae, Nitrospiraceae, Sphingomonadaceae, and Comamonadaceae decreased by $21.5-85.7 \%$ compared to those of maize cultivation. Conversely, the relative abundances of Acidobacteriaceae, Koribacteraceae, Solibacteraceae, Sinobacteraceae, and Xanthomonadaceae increased by 21.0-86.0\% (Fig. 7b).

The relative abundance of fungal groups changes in soils cultivated with American ginseng

Fungal taxa differed between the soils of American ginseng and maize at the levels of order and family (Fig. 8; Additional file 1: Table S6). Compared with soils used for maize cultivation, the relative abundances of Arthoniales, 

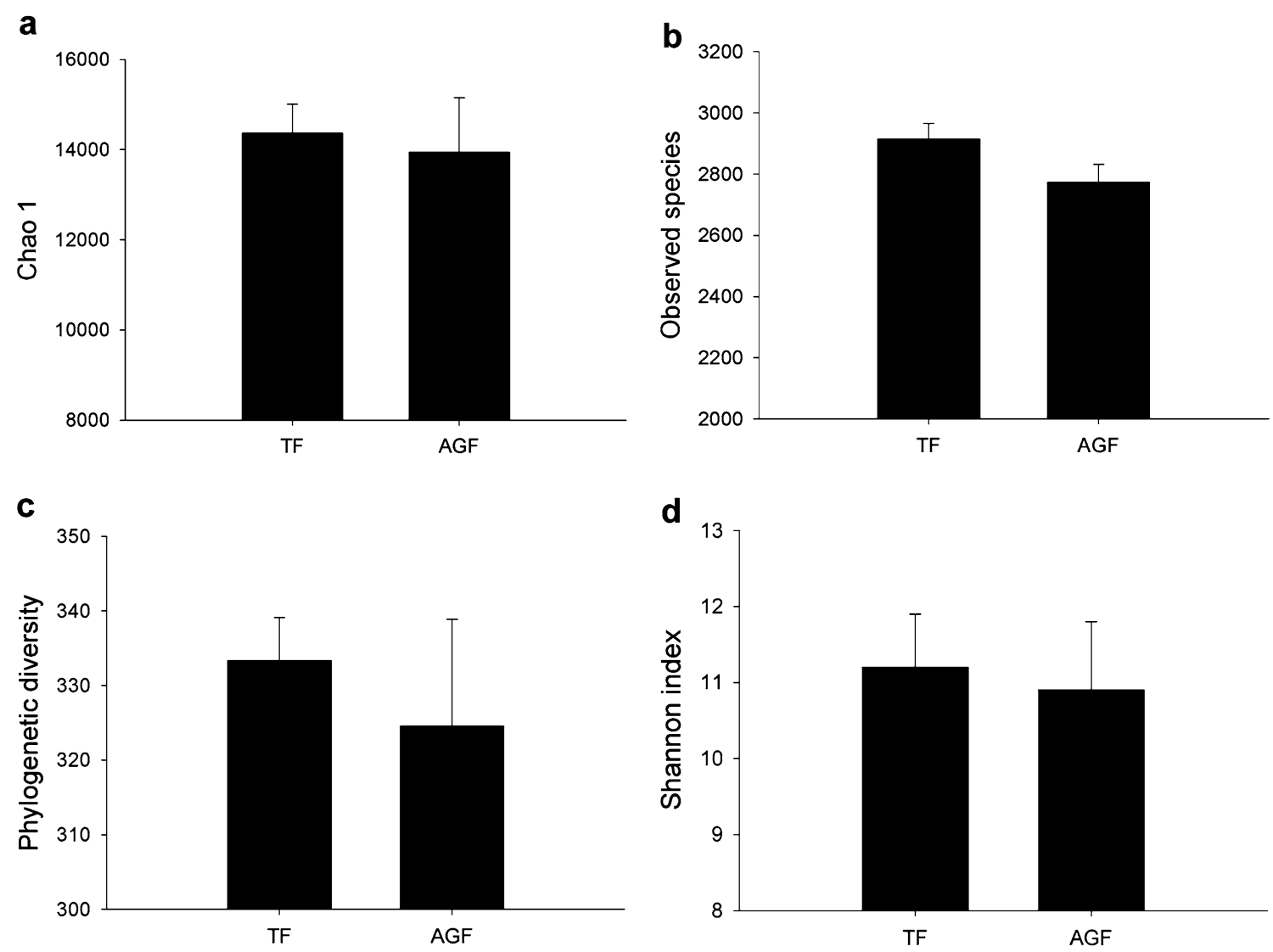

Fig. 3 Changes of Chao 1 (a), observed species (b), phylogenetic diversity (c), and Shannon index (d) for the 16S rRNA gene sequences from soils used for traditional crops and American ginseng. TF and AGF refer to soil samples from traditional farmland and American ginseng farmland, respectively. The value of each bar represents the mean $\pm S D$ of $n=3$

Pleosporales, Lecanorales, and Sordariales declined by 61.4-274\% in soils of American ginseng cultivation. In contrast, the relative abundances of Chaetothyriales, Eurotiales, Hypocreales, Microascales, Calosphaeriales, Xylariales, Helotiales, Pezizales, and Saccharomycetales increased by $13.4-132 \%$ in soils used for American ginseng cultivation (Fig. 8a). Furthermore, the relative abundances of Sclerotiniaceae, Pyronemataceae, Debaryomycetaceae, Cystofilobasidiaceae, Herpotrichiellaceae, Trichocomaceae, Bionectriaceae, Clavicipitaceae, Halosphaeriaceae, Calosphaeriaceae, and Amanitaceae increased by $28.1-215 \%$, and the relative abundances of Pleosporineae, Lecanorineae, Chaetomiaceae, Sordariaceae, and Amphisphaeriaceae showed declining trends $(0.6-58.5 \%)$ in soils of American ginseng cultivation compared with those of maize cultivation (Fig. 8b).

\section{Discussion}

The increase in the ratio of fungi to bacteria was observed in the soils of American ginseng cultivation compared to those of maize in our study. Many studies have reported that the ratios of fungi to bacteria were altered in cropping systems $[24,25]$. The ratio of fungi to bacteria significantly increased in the continuous cropping of $P$. notoginseng [13]. These conclusions are also supported by our results. The ratio of fungi to bacteria is the most important characteristic of soil functionality and can serve as an indicator of ecosystem processes [26]. These results could imply soil functionality was changed in a continuous cropping system.

Our results confirmed a decline in bacterial diversity and an increase in fungal diversity in soils subjected to a continuous cropping system of American ginseng. As mentioned above, phylotype diversity of fungi also increased during continuous cropping of peanut [5]. Benizri et al. [27] reported that the bacterial diversity changed in the continuous cropping soil of peaches. It has been proposed that the diversity of soil microorganisms is related to the maintenance of soil health and quality [8]. Fungal diversity was associated with the death rate of $P$. notoginseng in the continuous cropping system [13]. Fungal diversity has been suggested to have beneficial 

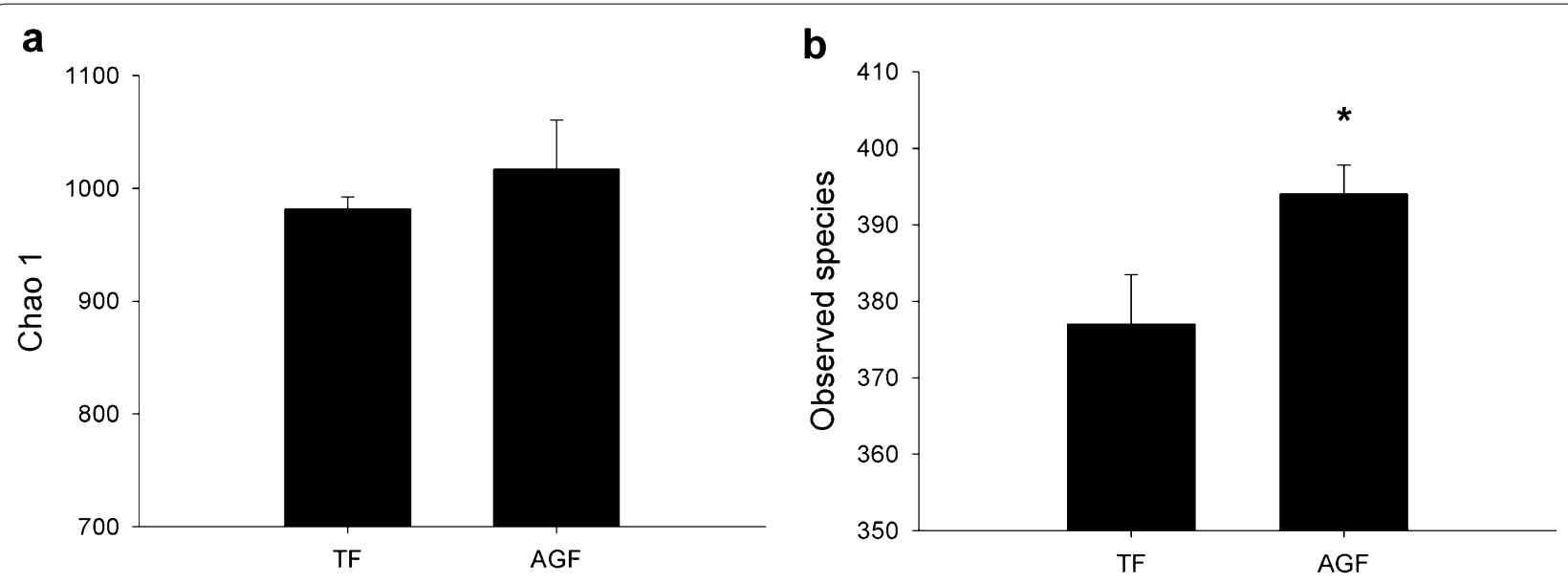

C

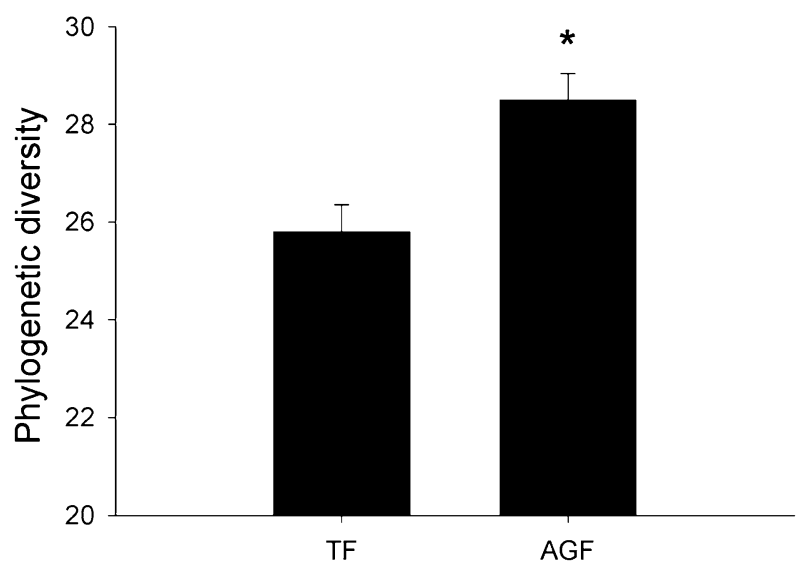

d

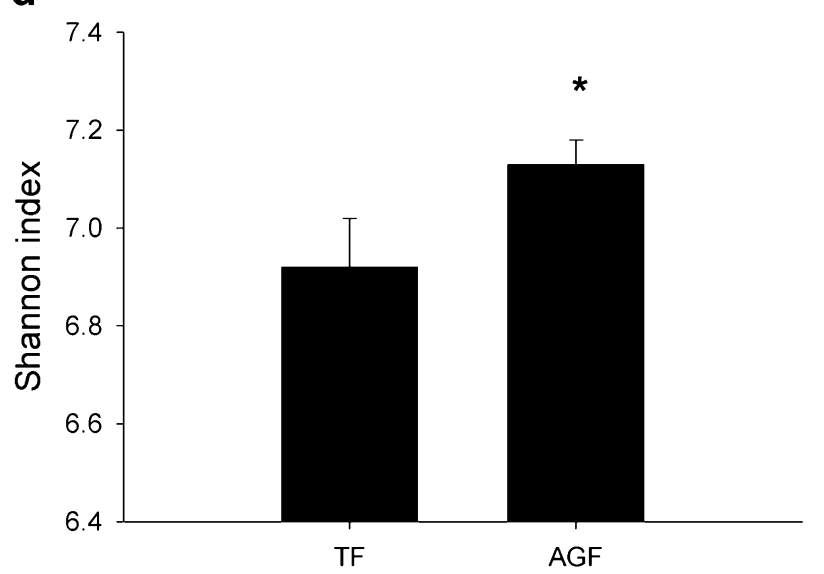

Fig. 4 Changes of Chao 1 (a), observed species (b), phylogenetic diversity (c), and Shannon index (d) for the 185 rRNA gene sequences from soils used for traditional crops and American ginseng. TF and AGF refer to soil samples from traditional farmland and American ginseng farmland, respectively. An asterisk denotes a significant difference between TF and AGF at $P<0.05$. The value of each bar represents the mean $\pm \operatorname{SD}$ of $n=3$

effects on plant production by suppressing pathogens [28]. A decrease in soil microbial diversity was responsible for the development of soil-borne plant diseases [29]. Taking the obtained findings together, the changes of microbial diversity in continuous cropping of American ginseng could alter the micro-ecological environment.

Cropping systems changed the composition of soil microorganisms and affected the soil health and quality $[11,30,31]$. Significant changes of microbial communities were observed in rhizosphere soils during the continuous cultivation of ginseng [32]. Fungal populations exhibited significant dynamic changes in the continuous cropping of peanut, and both pathogenic and beneficial fungi were positively selected over time [5]. Plant species is thought to select specific microbial populations in the rhizosphere and root exudates is a driving force $[8,33,34]$. American ginseng is a perennial plant and its root exudates accumulate in the rhizosphere, possibly presenting substrates for several groups of bacterial communities. For instance, Proteobacteria and Bacteroidetes increased in relative abundance in highnitrogen plots [6], which may explain the increase in the proportions of their abundances. However, plants do not only provide nutrients for microbial communities, but also contain a series of antimicrobial metabolites in their root exudates $[10,33,34]$. This may explain observed decreases in the relative abundances of microbial communities. Additionally, changes in soil chemical properties are likely to drive changes in microbial community composition [24]. Our analyses did not 

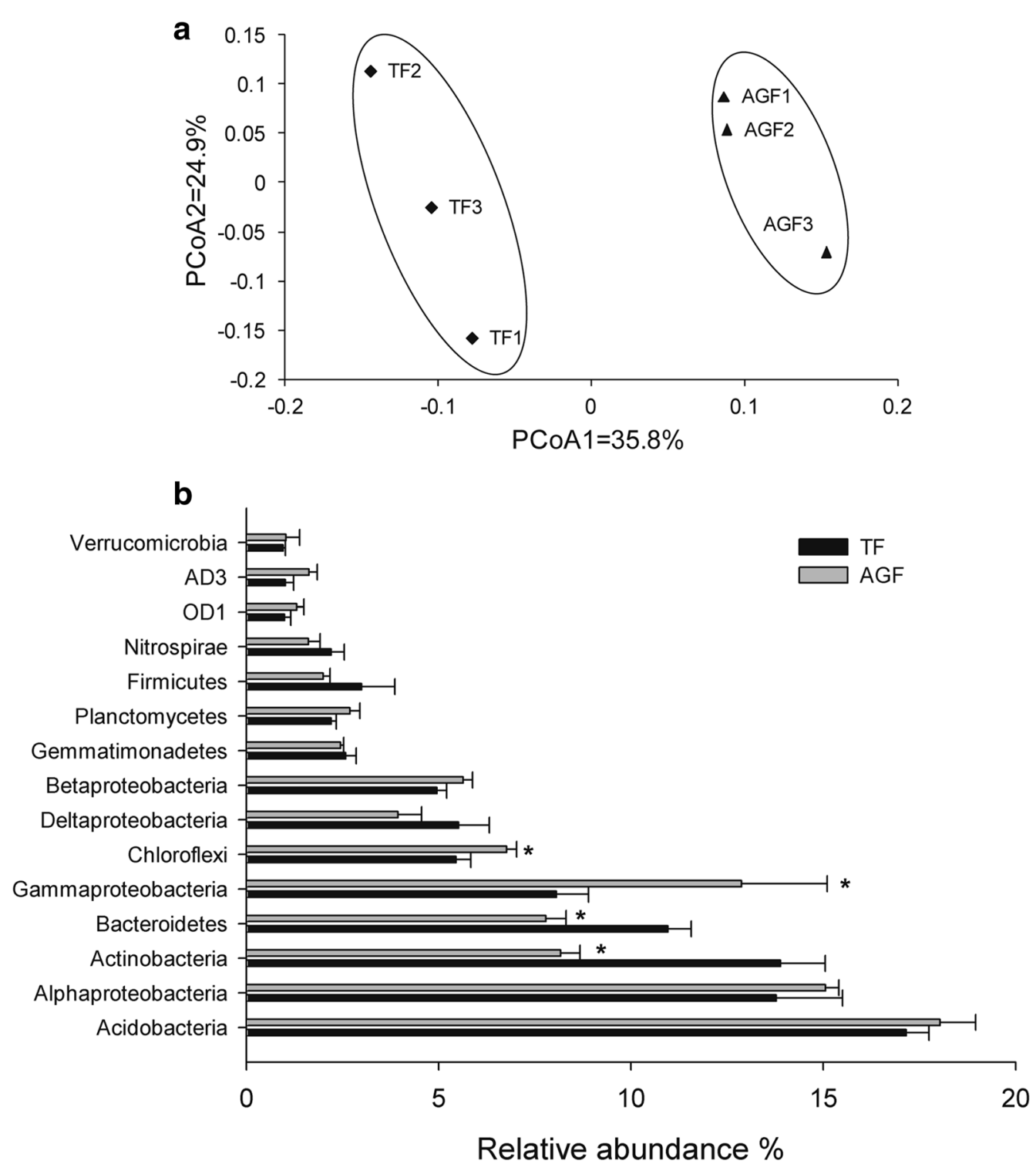

Fig. 5 Changes in the composition of the bacterial community in soils used for traditional crops and American ginseng. a PCoA ordination plots show the relatedness of samples that were separated using weighted UniFrac distances of classified 16S rRNA gene sequences. $\mathbf{b}$ The relative abundances of the bacterial community $(>1 \%)$ at the phylum level. TF and AGF refer to soil samples from traditional farmland and American ginseng farmland, respectively. An asterisk denotes a significant difference between TF and AGF at $P<0.05$. The value of each bar represents the mean \pm SD of $n=3$

show significant differences in $\mathrm{pH}$, total nitrogen, available potassium, or organic matter between the soils of traditional cropping and American ginseng continuous cropping (Additional file 1: Table S1). Additionally, the contents of Olsen-phosphatase were significant difference in soils of traditional cropping compared to those of American ginseng, which effects on the microbial communities would need to further verification. Thus, cropping system was speculated as a dominant factor in disrupting the balance of soil microbial communities [13]. Changes in the composition of bacterial community may lead to variations in metabolic capacity, biodegradation, and disease-suppression abilities [8, 35]. The negative impact of continuous cropping on 

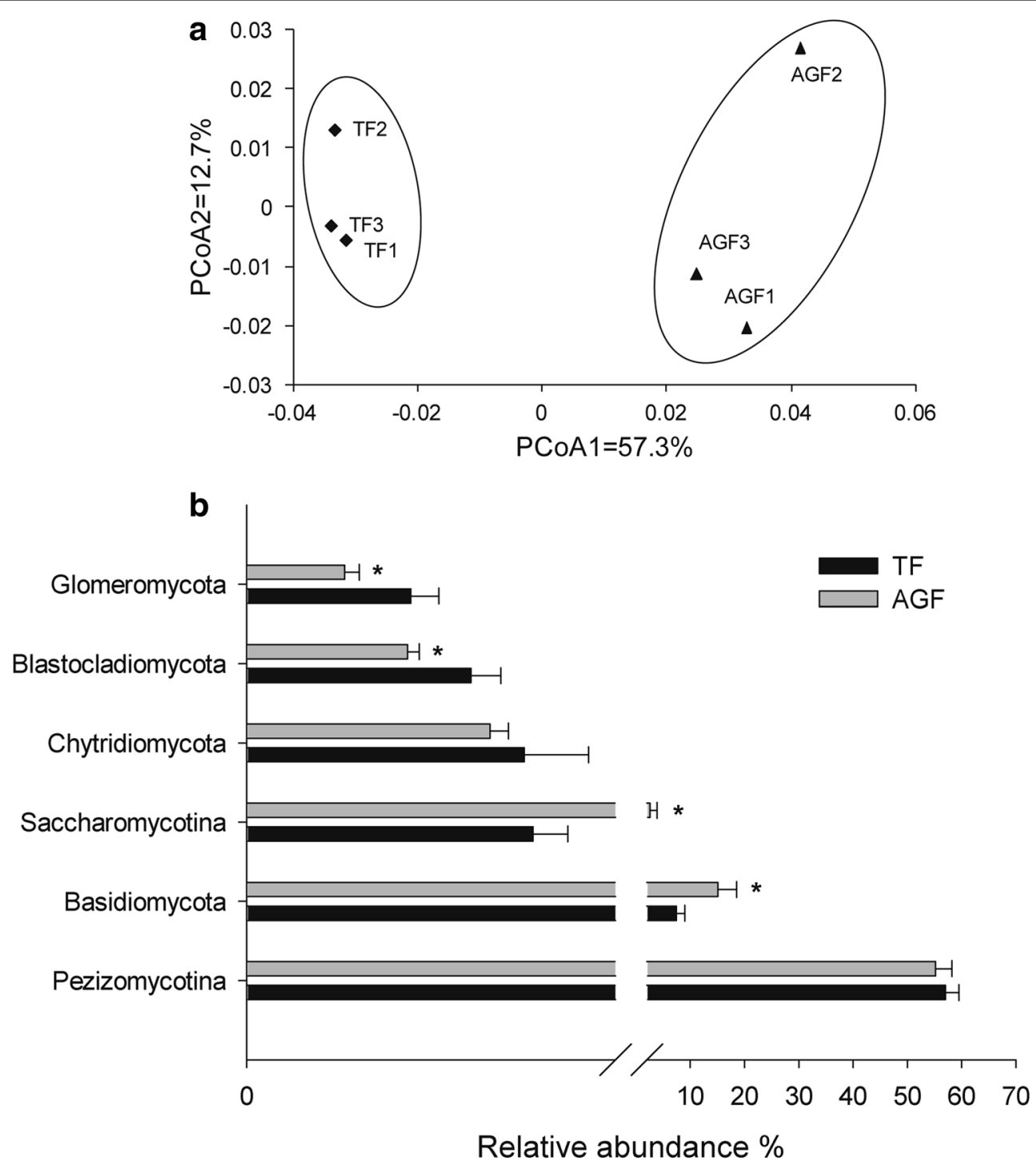

Fig. 6 Changes in the composition of the fungal community in soils of traditional farmland and American ginseng farmland. a PCoA ordination plots show the relatedness of samples that were separated using weighted UniFrac distances of classified $18 \mathrm{~S}$ rRNA gene sequences. b The relative abundances of the fungal community at the phylum level. TF and AGF refer to soil samples from traditional farmland and American ginseng farmland, respectively. An asterisk denotes a significant difference between TF and AGF at $P<0.05$. The value of each bar represents the mean \pm SD of $n=3$

soil productivity has been shown [36]. These findings indicate that continuous cropping might have negative effects on ecosystem processes that are central to key ecosystem services. In this study, high-throughput sequencing analyses provided simultaneous and detailed insight into the bacterial and fungal communities in the agro-ecosystem of medicinal plants. HerbGenomics has been provided as a new discipline and metagenomic analyses of soil microbial populations is an important part of this discipline [37].

\section{Conclusions}

In summary, compared to those of traditional cropping system, the bacterial diversity decreased and the fungal diversity showed increasing trends in soils used for the continuous cropping of American ginseng; meanwhile, the 


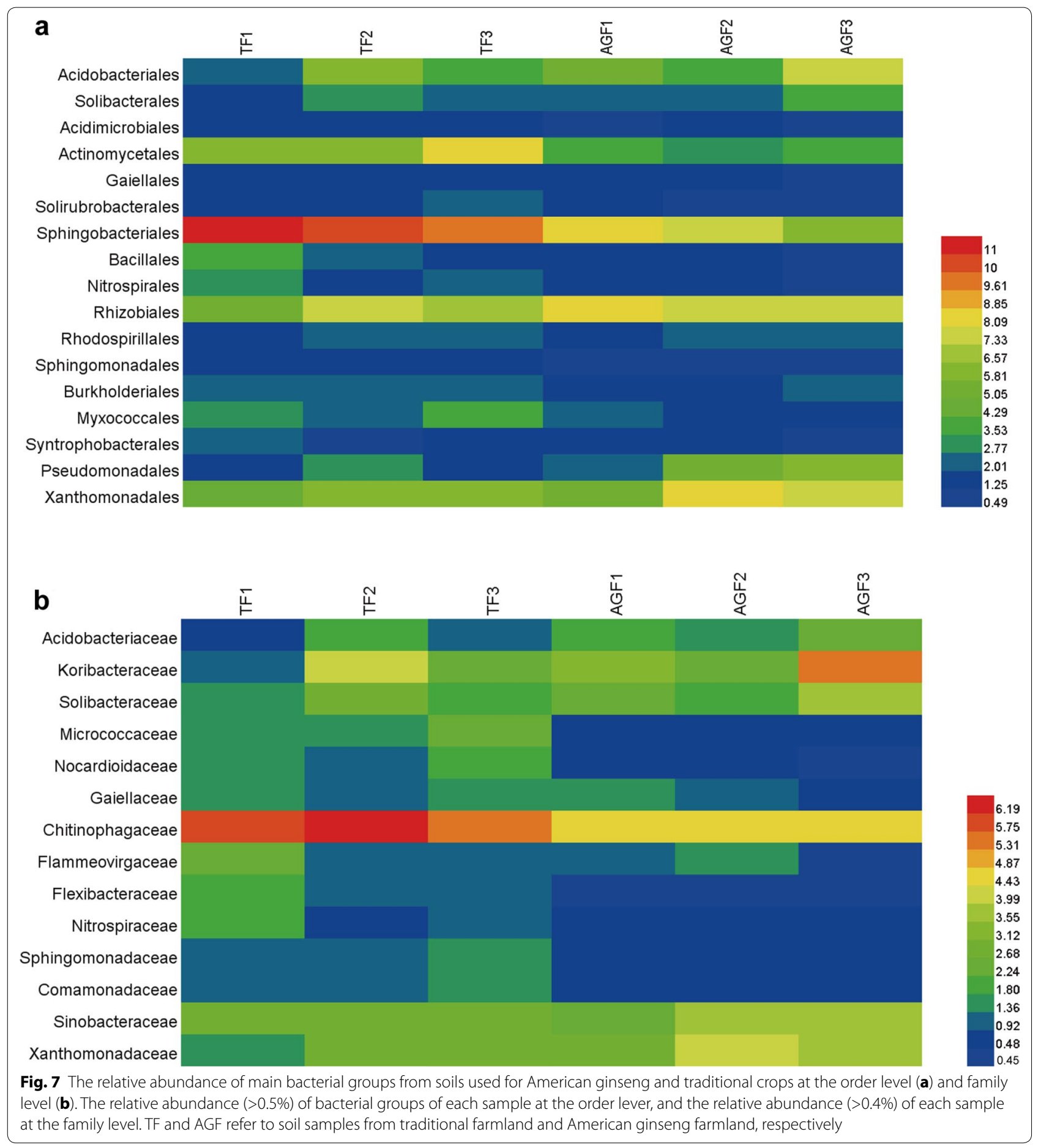

composition of bacterial and fungal communities changed in soils cultivated with American ginseng. Our work will be of great significance for the understanding of continuous cropping obstacles from rhizospheric microorganisms, promoting the continuous development of medicinal plants and accelerating the recycling of soil resources. 


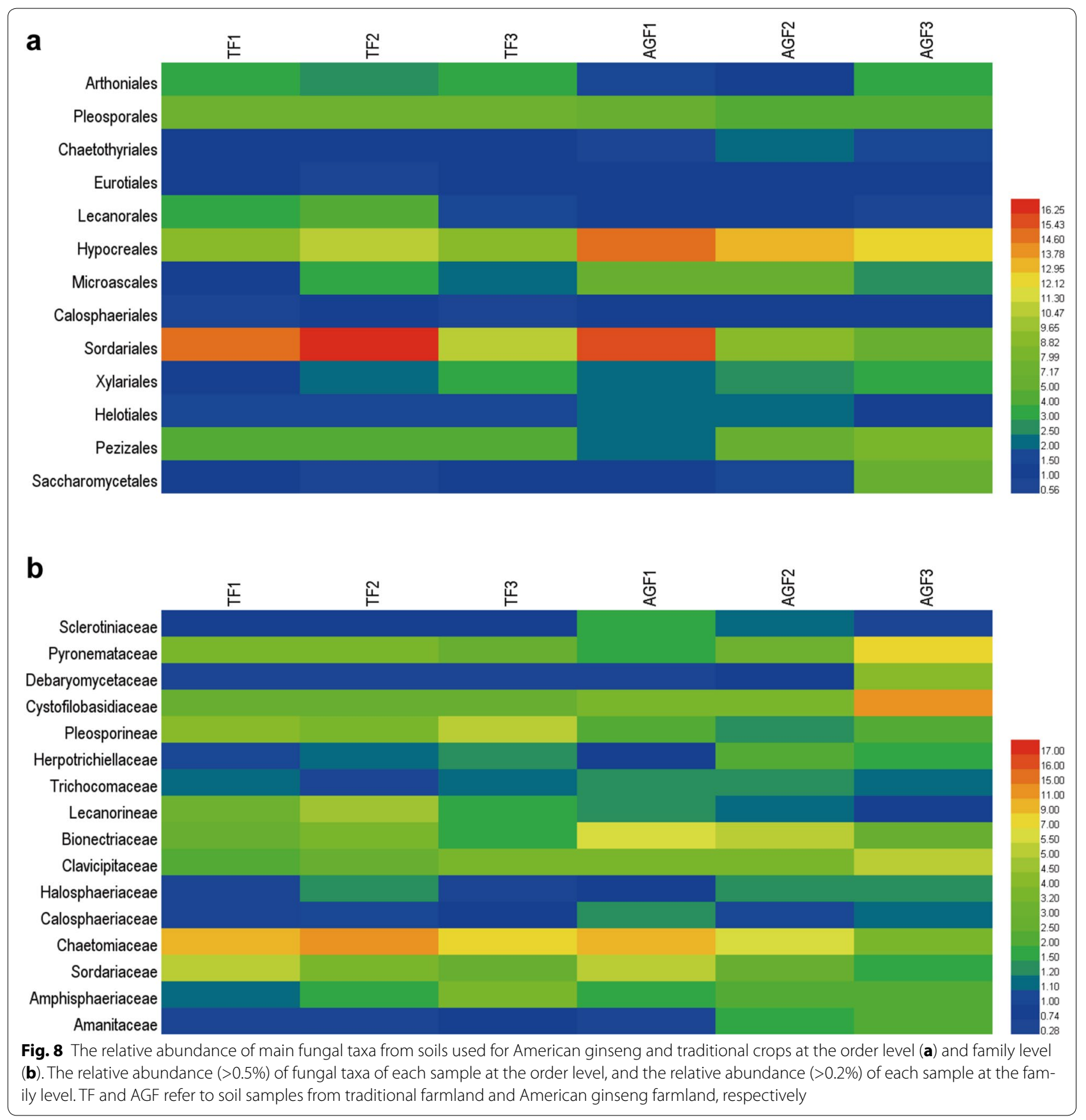

\section{Additional files}

Additional file 1: Table S1-S6. The supplementary information for this article.

Additional file 2. Minimum Standards of Reporting Checklist.

\section{Abbreviations}

TF: traditional farmland; AGF: American ginseng farmland; rRNA: ribosomal RNA; PCR: polymerase chain reaction; QPCR: quantitative polymerase chain reaction; PCOA: principal coordinates analysis; RDP: ribosomal database project; OTUs: operational taxonomic units.

\section{Authors' contributions}

LD carried out the molecular experiments, analyzed the data and wrote the manuscript. JX and GW carried out the data analyses. LZ performed the 
molecular experiments. JY and XL contributed to the field experiments and collected samples. SC conceived of the study, contributed to the design and interpretation of the research. All authors read and approved the final manuscript.

\section{Acknowledgements}

We thank Prof. Yujun Zhang, a member of Institute of Chinese Materia Medica, China Academy of Chinese Medical Sciences, contributed to the modification of manuscript.

\section{Competing interests}

All authors declare that they have no competing interests.

\section{Availability of data and materials}

The dataset supporting the conclusions of this article is included within the article and its Additional files 1, 2

\section{Funding}

This study was supported by grants from National Science Foundation of China (Nos. 81603238 and 81403053).

\section{Publisher's Note}

Springer Nature remains neutral with regard to jurisdictional claims in published maps and institutional affiliations.

Received: 23 April 2017 Accepted: 22 June 2017

Published online: 03 July 2017

\section{References}

1. Sen S, Chen S, Feng B, Wu Y, Lui Y, Chakrabart S. Preventive effects of North American ginseng (Panax quinquefolium) on diabetic nephropathy. Phytomedicine. 2012;19:494-505.

2. He CN, Gao WW, Yang JX, Bi W, Zhang XS, Zhao YJ. Identification of autotoxic compounds from fibrous roots of Panax quinquefolium L. Plant Soil. 2009:318:63-72.

3. Ogweno JQ, Yu JQ. Autotoxic potential in soil sickness: a re-examination. Allelopathy J. 2006;18:93-101.

4. Huang LF, Song LX, Xia XJ, Mao WH, Shi K, Zhou YH, Yu JQ. Plant-Soil feedbacks and soil sickness: from mechanisms to application in agriculture. J Chem Ecol. 2013;39:232-42.

5. Chen M, Li X, Yang Q, Chi X, Pan L, Chen N, Yang Z, Wang T, Wang M, Yu S. Soil eukaryotic microorganism succession as affected by continuous cropping of peanut-pathogenic and beneficial fungi were selected. PLOS ONE. 2012;7:e40659.

6. Fierer N, Lauber CL, Ramirez KS, Zaneveld J, Bradford MA, Knight R. Comparative metagenomic, phylogenetic and physiological analyses of soil microbial communities across nitrogen gradients. ISME J. 2012;6:1007-17.

7. Anderson TH. Microbial eco-physiological indicators to assess soil quality. Agr Ecosyst Environ. 2003;98:285-93.

8. Garbeva P, van Veen JA, van Elsas JD. Microbial diversity in soil: selection of microbial populations by plant and soil type and implications for disease suppressiveness. Annu Rev Phytopathol. 2004;42:243-70.

9. Green JL, Bohannan BJ, Whitaker RJ. Microbial biogeography: from taxonomy to traits. Science. 2008;320:1039-43.

10. Berg G, Smalla K. Plant species and soil type cooperatively shape the structure and function of microbial communities in the rhizosphere. FEMS Microbiol Ecol. 2009;68:1-13.

11. Li C, Li X, Kong W, Wu Y, Wang J. Effect of monoculture soybean on soil microbial community in the Northeast China. Plant Soil. 2010;330:423-33.

12. Qi J, Zhao X, Zhou L, Sun P, Zhang X, Li X. Soil microbial community composition and diversity in Panax quinquefolius rhizosphere. China J Chin Mater Med. 2010;35(18):2378-82.

13. Dong L, Xu J, Feng G, Li X, Chen S. Soil bacterial and fungal community dynamics in relation to Panax notoginseng death rate in a continuous cropping system. Sci Rep. 2016;6:31802.
14. Heuberger H, Bauer R, Friedl F, Heubl G, Hummelsberger J, Nögel R, Seidenberger R, Torres-Londoño P. Cultivation and breeding of Chinese medicinal plants in Germany. Planta Med. 2010;76:1956-62.

15. Zhang B, Peng Y, Zhang Z, Liu H, Qi Y, Liu S, Xiao P. GAP production of TCM herbs in China. Planta Med. 2010;76:1948-55.

16. Wu L, Wang H, Zhang Z, Lin R, Zhang Z, Lin W. Comparative metaproteomic analysis on consecutively Rehmann ia g/utinosa-monocultured rhizosphere soil. PLoS ONE. 2011;6:e20611.

17. Fierer N, Jackson JA, Vilgalys R, Jackson RB. Assessment of soil microbial community structure by use of taxon-specific quantitative PCR assays. Appl Environ Microbiol. 2005:71:4117-20.

18. Rousk J, Bååth E, Brookes PC, Lauber CL, Lozupone C, Caporaso JG, Knight $\mathrm{R}$, Fierer N. Soil bacterial and fungal communities across a pH gradient in an arable soil. ISME J. 2010:4:1-12.

19. Caporaso JG, Bittinger K, Bushman FD, DeSantis TZ, Andersen GL, Knight R. PyNAST: a flexible tool for aligning sequences to a template alignment. Bioinformatics. 2010;26:266-7.

20. Caporaso JG, Kuczynski J, Stombaugh J, Bittinger K, Bushman FD, Costello EK, Fierer N, Peña AG, Goodrich JK. QIIME allows analysis of high-throughput community sequencing data. Nat Methods. 2010;7:335-6.

21. Price MN, Dehal PS, Arkin AP. FastTree: computing large minimum evolution trees with profiles instead of a distance matrix. Mol Biol Evol. 2009;26:1641-50.

22. Wang Q, Garrity GM, Tiedje JM, Cole JR. Naïve Bayesian classifier for rapid assignment of rRNA sequences into the new bacterial taxonomy. Appl Environ Microbiol. 2007;73:5261-7.

23. Quast C, Pruesse E, Yilmaz P, Gerken J, Schweer T, Yarza P, Peplies J. The SILVA ribosomal RNA gene database project: improved data processing and Web-based tools. Nucleic Acids Res. 2013:41:D590-6.

24. Lauber CL, Strickland MS, Bradford MA, Fierer N. The influence of soil properties on the structure of bacterial and fungal communities across land-use types. Soil Biol Biochem. 2008;40:2407-15.

25. e Silva MCP, Dias AC, van Elsas JD, Salles JF. Spatial and temporal variation of archaeal, bacterial and fungal communities in agricultural soils. PLoS ONE. 2012;7:e51554.

26. van der Heijden MG, Bardgett RD, van Straalen NM. The unseen majority: soil microbes as drivers of plant diversity and productivity in terrestrial ecosystems. Ecol Lett. 2008;11:296-310.

27. Benizri E, Piutti S, Verger S, Pagès L, Vercambre G, Poessel JL. Replant diseases: bacterial community structure and diversity in peach rhizosphere as determined by metabolic and genetic fingerprinting. Soil Biol Biochem. 2005:37:1738-46.

28. Wehner J, Antunes PM, Powell JR, Mazukatow J, Rillig MC. Plant pathogen protection by arbuscular mycorrhizas: a role for fungal diversity? Pedobiologia. 2010;53:197-201.

29. Mazzola M. Assessment and management of soil community structure for disease suppression. Annu Rev Phytopathol. 2004;2004(42):35-59.

30. Acosta-Martínez V, Burow G, Zobeck TM, Allen VG. Soil microbial communities and function in alternative systems to continuous cotton. Soil Sci Soc Am J. 2010;74:1181-92.

31. Sanguin H, Sarniguet A, Gazengel K, Moënne-Loccoz Y, Grundmann GL. Rhizosphere bacterial communities associated with disease suppressiveness stages of take-all decline in wheat monoculture. New Phytol. 2009;184:694-707.

32. Ying Y, Ding W, Zhou Y, Li Y. Influence of Panax ginseng continuous cropping on metabolic function of soil microbial communities. Chin Herb Med. 2012:4:329-34.

33. Bais HP, Weir TL, Perry LG, Gilroy S, Vivanco JM. The role of root exudates in rhizosphere interactions with plants and other organisms. Annu Rev Plant Biol. 2006;57:234-66.

34. Mazzola M, Manici LM. Apple replant disease: role of microbial ecology in cause and control. Annu Rev Phytopathol. 2012;50:45-65.

35. Bell TH, Yergeau E, Maynard C, Juck D, Whyte LG, Gree CW. Predictable bacterial composition and hydrocarbon degradation in Arctic soils following diesel and nutrient disturbance. ISME J. 2013;7:1200-10.

36. Nayyar A, Hamel C, Lafond G, Gossen BD, Hanson K, Germida J. Soil microbial quality associated with yield reduction in continuous-pea. Appl Soil Ecol. 2009:43:115-21.

37. Chen S, Song J. HerbGenomics. China J Chin Mater Med. 2016:41:3381-91 